\title{
Intraosseous Hibernoma: A Rare and Unique Intraosseous Lesion
}

\author{
Boram Song ${ }^{1} \cdot$ Hye Jin Ryu ${ }^{2}$ \\ Cheol Lee ${ }^{1} \cdot$ Kyung Chul Moon ${ }^{1,3}$ \\ Departments of ${ }^{1}$ Pathology and ${ }^{2}$ Radiology, \\ Seoul National University College of Medicine \\ Seoul; ${ }^{3}$ Kidney Research Institute, Medical \\ Research Center, Seoul National University \\ College of Medicine, Seoul, Korea
}

Received: July 4, 2017

Revised: July 26, 2017

Accepted: July 27, 2017

Corresponding Author

Kyung Chul Moon, MD

Department of Pathology, Kidney Research Institute, Medical Research Center, Seoul National University College of Medicine, 103 Daehak-ro, Jongno-gu, Seoul 03080, Korea

Tel: +82-2-2072-1767

Fax: +82-2-743-5530

E-mail: blue7270@snu.ac.kr

\begin{abstract}
Background: Hibernoma is a rare benign tumor of adults that is composed of multivacuolated adipocytes resembling brown fat cells. Hibernoma typically occurs in soft tissue, and intraosseous examples are very rare. Intraosseous hibernomas can radiologically mimic metastatic carcinoma and other tumorous conditions. Methods: To collect the intraosseous hibernomas, we searched the pathologic database and reviewed the hematoxylin and eosin (H\&E)-stained slides of bone biopsy samples performed to differentiate radiologically abnormal bone lesions from 2006 to 2016. A total of six intraosseous hibernoma cases were collected, and clinical and radiological information was verified from electronic medical records. H\&E slide review and immunohistochemical staining for CD68, pan-cytokeratin, and S-100 protein were performed. Results: Magnetic resonance imaging of intraosseous hibernomas showed low signal intensity with slightly hyperintense foci on T1 and intermediate to high signal intensity on T2 weighted images. Intraosseous hibernomas appeared as heterogeneous sclerotic lesions with trabecular thickening on computed tomography scans and revealed mild hypermetabolism on positron emission tomography scans. Histopathologically, the bone marrow space was replaced by sheets of multivacuolated, foamy adipocytes resembling brown fat cells, without destruction of bone trabeculae. In immunohistochemical analysis, the tumor cells were negative for CD68 and pan-cytokeratin and positive for S-100 protein. Conclusions: Intraosseous hibernoma is very rare. This tumor can be overlooked due to its rarity and resemblance to bone marrow fat. Pathologists need to be aware of this entity to avoid misdiagnosis of this rare lesion.
\end{abstract}

Key Words: Hibernoma; Bone neoplasms; Pathology; Immunohistochemistry
Hibernoma is a rare benign adipocytic tumor of brown fat. ${ }^{1}$ This tumor was originally described as a "pseudolipoma" by Merkel in 1906. In 1914, Gery ${ }^{3}$ noticed a histological resemblance to the brown fat in hibernating animals and renamed the tumor "hibernoma". The typical histologic feature of the tumor is multivacuolated adipocytes with centrally located nuclei resembling a brown fat cell. ${ }^{1}$

Hibernomas commonly occur in the soft tissue of the thigh, followed by the shoulder, back, and neck. ${ }^{1}$ Intraosseous location has been rarely reported. Less than 20 cases are currently reported in the English literature. ${ }^{4-13}$ Radiologically, intraosseous hibernomas can mimic metastatic carcinoma and other bone lesions, such as a hemangioma, bone island, or benign notochordal lesion. ${ }^{5,8,10}$ Only bone biopsy and pathologic diagnosis can distinguish intraosseous hibernomas from other common conditions. In this study, we present six cases of intraosseous hibernomas with clinical, radiologic, and pathological findings.

\section{MATERIALS AND METHODS}

\section{Cases and clinicopathologic information}

The pathologic database of the Department of Pathology, Seoul National University Hospital, from 2006 to 2016 was searched with the keywords of hibernoma or brown fat, and three intraosseous hibernomas were found.

We also reviewed the hematoxylin and eosin (H\&E)-stained slides of bone biopsy cases with radiologic and clinical impressions of bone marrow involvement of metastatic carcinoma, lymphoma, chronic osteomyelitis, and hemangioma cases between 2006 and 2016, and three additional intraosseous hibernoma cases were newly identified.

A total of six cases were included in this study. In all cases, the bone biopsy was performed with a 14-gauge core needle under computed tomography (CT) or fluoroscopy guidance. We reevaluated the $\mathrm{H} \& \mathrm{E}$ slides to confirm the adequacy of the initial diagnosis and to analyze the various pathological features. Imaging studies were also reviewed by the musculoskeletal radiologist. The corresponding clinical data were obtained from the patient's 
medical records.

This study was approved by the Institutional Review Board (IRB) of Seoul National University Hospital (H-1611-004-803). The informed consent was waived by IRB.

\section{Immunohistochemical staining}

Immunohistochemical (IHC) staining for S-100 protein (Dako, Santa Clara, CA, USA), cytokeratin (Dako) and CD68 (Dako) was performed on formalin-fixed, paraffin-embedded tissue for all cases to validate the diagnosis of hibernoma. All IHC analyses were performed using the Ventana Benchmark XT automated staining system (Ventana Medical Systems, Tucson, AZ, USA).

\section{RESULTS}

\section{Clinical and radiologic features}

The clinical and radiologic features of the six patients are summarized in Table 1. The patients' ages ranged from 45 to 71 years. Three patients were male, and three were female. Five of six cases occurred in the axial skeleton; two in a thoracic vertebral body, two in a lumbar vertebral body, and one in the sacrum. The other case presented in the distal femur. Five of six patients initially presented with musculoskeletal pain (low back pain in four and knee pain in one patient), and the intraosseous hibernomas were identified during the diagnostic work-up to determine the cause of pain. The remaining case was asymptomatic and detected incidentally during preoperative work-up for a hepatocellular carcinoma. In three patients presenting with low back pain, imaging studies revealed disc bulging and spinal canal stenosis in addition to the bone lesion at the pain site. The remaining two patients complaining of pain revealed intramedullary bone lesions only; however, the pain of these two patients was controlled by analgesics without additional intervention.

In all cases, magnetic resonance imaging (MRI) consistently showed hypointensity intermingled with slightly hyperintense foci on T1-weighted images and intermediate to high signal intensity on T2-weighted images (Fig. 1A, B). CT mainly demonstrated mild osteosclerosis (Fig. 1C). Positron emission tomography (PET) scanning was performed in one patient and revealed mild hypermetabolism in the lesion, supporting the suspicion of malignancy (Fig. 1D). A bone scan was performed in one hepatocellular carcinoma patient as part of a liver transplantation work-up and showed increased uptake mimicking metastatic carcinoma. Involvement of cortical bone and extraosseous mass formation was not present. Radiologic impressions of the lesions were reported as intraosseous hemangioma, bone marrow involvement of lymphoma, metastatic carcinoma, or osteomyelitis.

\section{Histological and IHC findings}

Histopathological examination of the core biopsy showed aggregates of large polygonal cells with finely vacuolated voluminous cytoplasm resembling brown fat cell (Fig. 2A). The multivacuolated fat cells were arranged in sheets and clusters or scattered singularly intermingled with fatty and hematopoietic marrow elements (Fig. 2B). They had small, centrally located nuclei indented by vacuolated cytoplasm. Neither mitotic figures nor nuclear atypia were identified. The tumor cells infiltrated and replaced marrow space between the bony trabeculae without destroying the anatomy, although lamellar bony trabeculae within the lesion were slightly hypertrophied and showed mild sclerosis (Fig. 2C). The lesions also had small to medium sized blood vessels inside (Fig. 2D). Hibernoma cells with vacuolated cytoplasm may look

Table 1. Clinical and radiologic data

\begin{tabular}{|c|c|c|c|c|c|c|}
\hline Case & Age (yr) & Sex & Reason for investigation & Site & Radiologic finding & Radiologic impression \\
\hline 1 & 71 & $\mathrm{~F}$ & Low back pain & L3 VB & $\begin{array}{l}\text { MR: T1, low, heterogenous; T2, high } \\
\text { CT: sclerosis } \\
\text { PET: mild hypermetabolism }\end{array}$ & $\begin{array}{l}\text { Metastasis } \\
\text { Lymphoma } \\
\text { Osteomyelitis } \\
\text { Hemangioma }\end{array}$ \\
\hline 2 & 49 & M & Low back pain & T12 VB & $\begin{array}{l}\text { MR: T1, low, heterogenous; T2, high } \\
\text { Simple X-ray: sclerosis }\end{array}$ & $\begin{array}{l}\text { Metastasis } \\
\text { Hemangioma }\end{array}$ \\
\hline 3 & 65 & M & Hepatocellular carcinoma & T12 VB & $\begin{array}{l}\text { MR: T1, low, heterogenous; T2, high } \\
\text { CT: sclerosis } \\
\text { Bone scan: increased uptake }\end{array}$ & Metastasis \\
\hline 4 & 68 & M & Low back pain & Sacral ala & $\begin{array}{l}\text { MR: T1, low, heterogenous; T2, high } \\
\text { CT: osteolysis with peripheral sclerosis }\end{array}$ & Hemangioma \\
\hline 5 & 45 & $\mathrm{~F}$ & Knee pain & Distal femur & $\begin{array}{l}\text { MR: T1, low, heterogenous; T2, high } \\
\text { CT: mild sclerosis }\end{array}$ & $\begin{array}{l}\text { Hemangioma } \\
\text { Lymphoma } \\
\text { Osteomyelitis }\end{array}$ \\
\hline
\end{tabular}

F, female; VB, vertebral body; MR, magnetic resonance; CT, computed tomography; PET, positron emission tomography; M, male. 
like lipoblasts or foamy histiocytes. Among our cases, one was initially misdiagnosed as lipoblast in marrow space, and another was misinterpreted as a foamy histiocyte collection.

With IHC staining, the vacuolated cells were positive for S-100 protein in nuclei and cytoplasm and negative for CD68 and pan-cytokeratin (AE1/AE3) (Fig. 3). These IHC findings were consistent with intraosseous hibernoma and excluded histiocytic lesion or hidden metastatic carcinoma as possible diagnoses.

\section{DISCUSSION}

Hibernoma is a rare benign tumor composed of brown fat. ${ }^{1}$
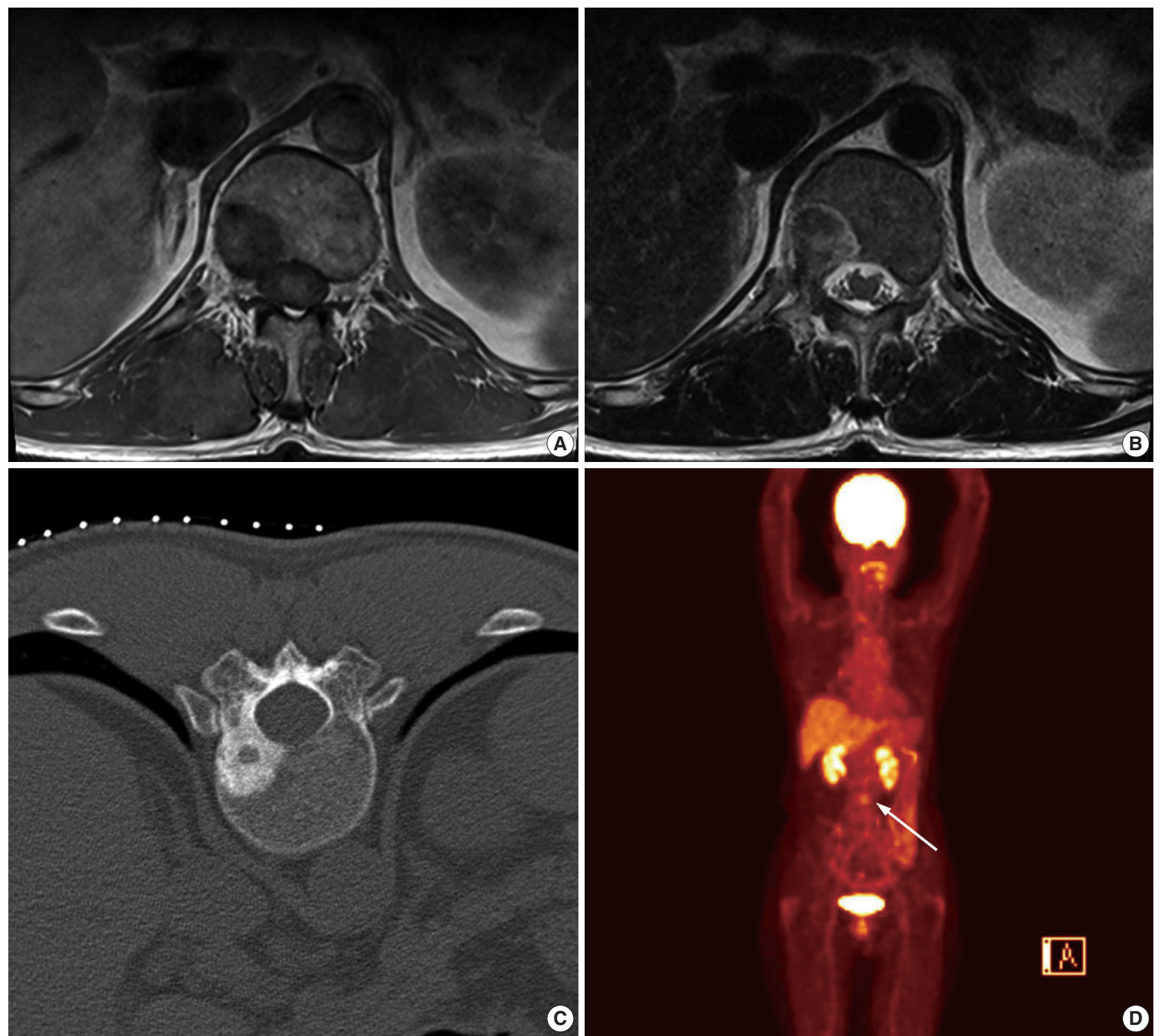

Brown fat is known to exist in restricted portions of the body of newborn humans and hibernating animals, performing a role in thermogenesis without shivering. Brown fat gradually disappears postnatally within a few years ${ }^{14,15}$ but can remain focally in the paravertebral and neck region, mediastinum, and retroperitoneum in adults. ${ }^{1,15}$

In the study of a large series of hibernomas from the Armed Forces Institute of Pathology (AFIP), USA, soft tissue hibernomas were reported to usually occur in 30- to 40-year-old adults, varying from 2 to 72 years of age, with almost equal distribution between the sexes. These tumors most commonly occur in the subcutaneous or intramuscular region of the thigh, which is not the normal

Fig. 1. Radiologic findings of intraosseous hibernoma. (A) Low signal intensity on T1-weighted magnetic resonance imaging (MRI). (B) Heterogeneous T2 high signal intensity on T2-weighted MRI. (C) Sclerotic change on computed tomography. (D) Mild hypermetabolism on positron emission tomography scan (arrow). 

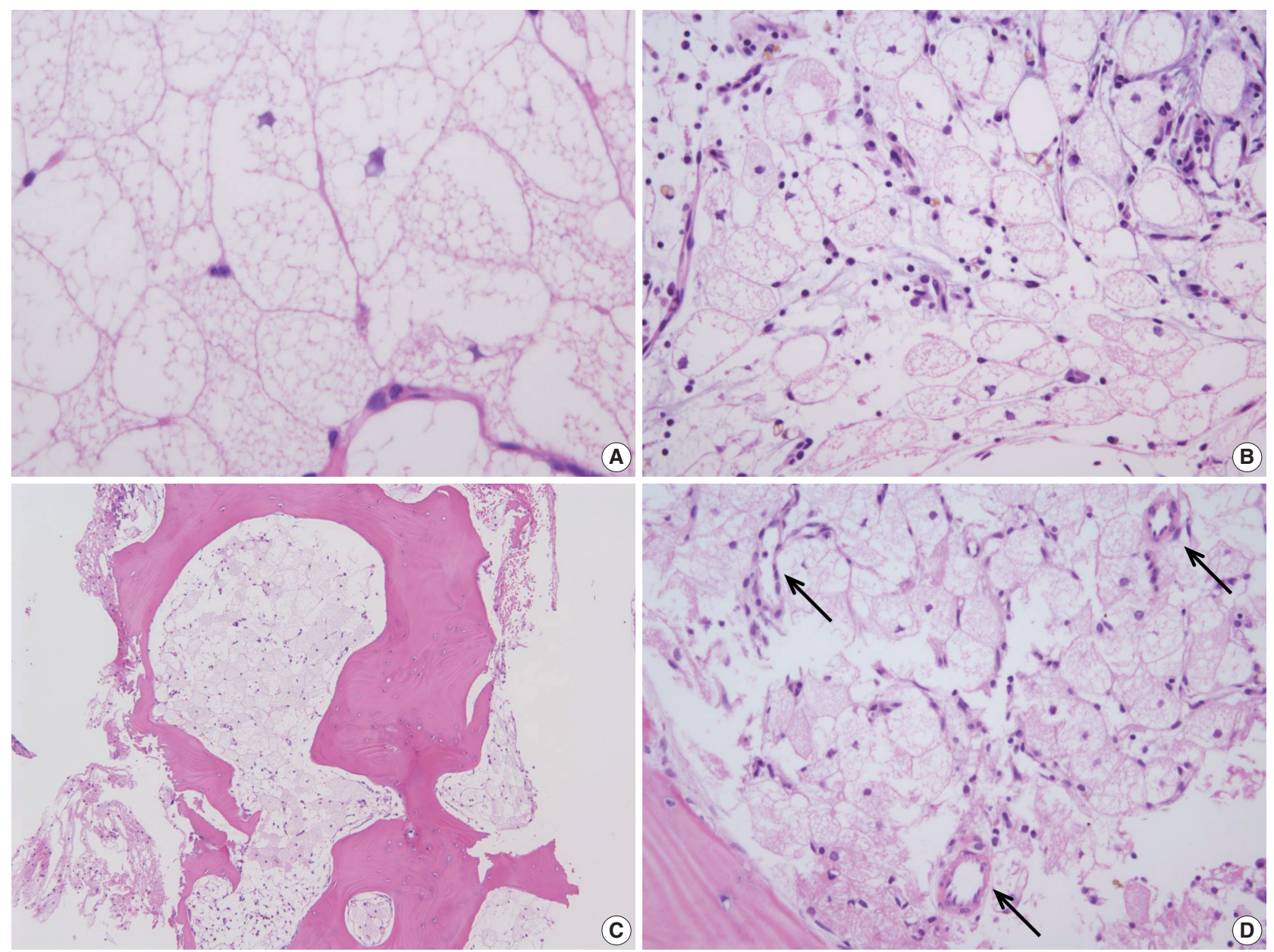

Fig. 2. Pathologic features of intraosseous hibernoma. (A) Brown fat cells with multivacuolated or granular cytoplasm indenting centrally located small nuclei. (B) Brown fat cells are intermingled with hematopoietic cells. (C) Bony trabeculae shows mild sclerosis. (D) Small to medium sized vessels (arrows) within the lesion.

location of brown fat. ${ }^{1}$

Hibernomas are very rarely found in an intraosseous location, with a total of 12 cases reported in the English literature up to now. ${ }^{4-8,10}$ Previous study of intraosseous hibernomas described slight female predominance and an age range of 40 to 85 years. ${ }^{10}$ In this study, we presented six additional cases of intraosseous hibernomas diagnosed at Seoul National University Hospital between 2006 and 2016. Our cohort showed similar sex and age distribution to the previous study, with three males and three females varying in ages from 45 to 71 years. Most of our cases (five of six patients) initially presented with musculoskeletal pain and underwent imaging work-up to determine the cause. Detected bone lesions were generally accompanied by disc herniation or spinal stenosis at the pain site. This finding had suggested that musculoskeletal pain, which had led the patient to seek medical attention, may be irrelevant to the bone lesion. To date, intraosseous hibernomas are usually considered to be asymp- tomatic. ${ }^{10}$ Only one previous case was reported symptomatic due to the resolution of symptoms after radioablation therapy. ${ }^{9}$ In our cohort, two of five patients presenting with pain had no other lesions that could cause pain besides the intraosseous lesion, allowing the possibility of intraosseous hibernoma-induced bone pain. However, the patients' pain was controlled by analgesics without additional interventions such as radioablation or curettage, suggesting that the pain was not actually caused by the intraosseous hibernoma.

Most reported intraosseous hibernomas were located in the axial skeleton, ${ }^{4-13}$ and five of our cases were also found in the axial skeleton. However, one case in our study was found in the distal femur and presented with leg pain. Radiologic features of the lesions were consistent with those described in the previous studies. ${ }^{5,7,8,10}$ Intraosseous hibernomas appeared as a sclerotic lesion on CT, with MRI showing T1 hypointensity with internal hyperintense foci, $\mathrm{T} 2$ heterogenous hyperintensity, and moderate contrast 

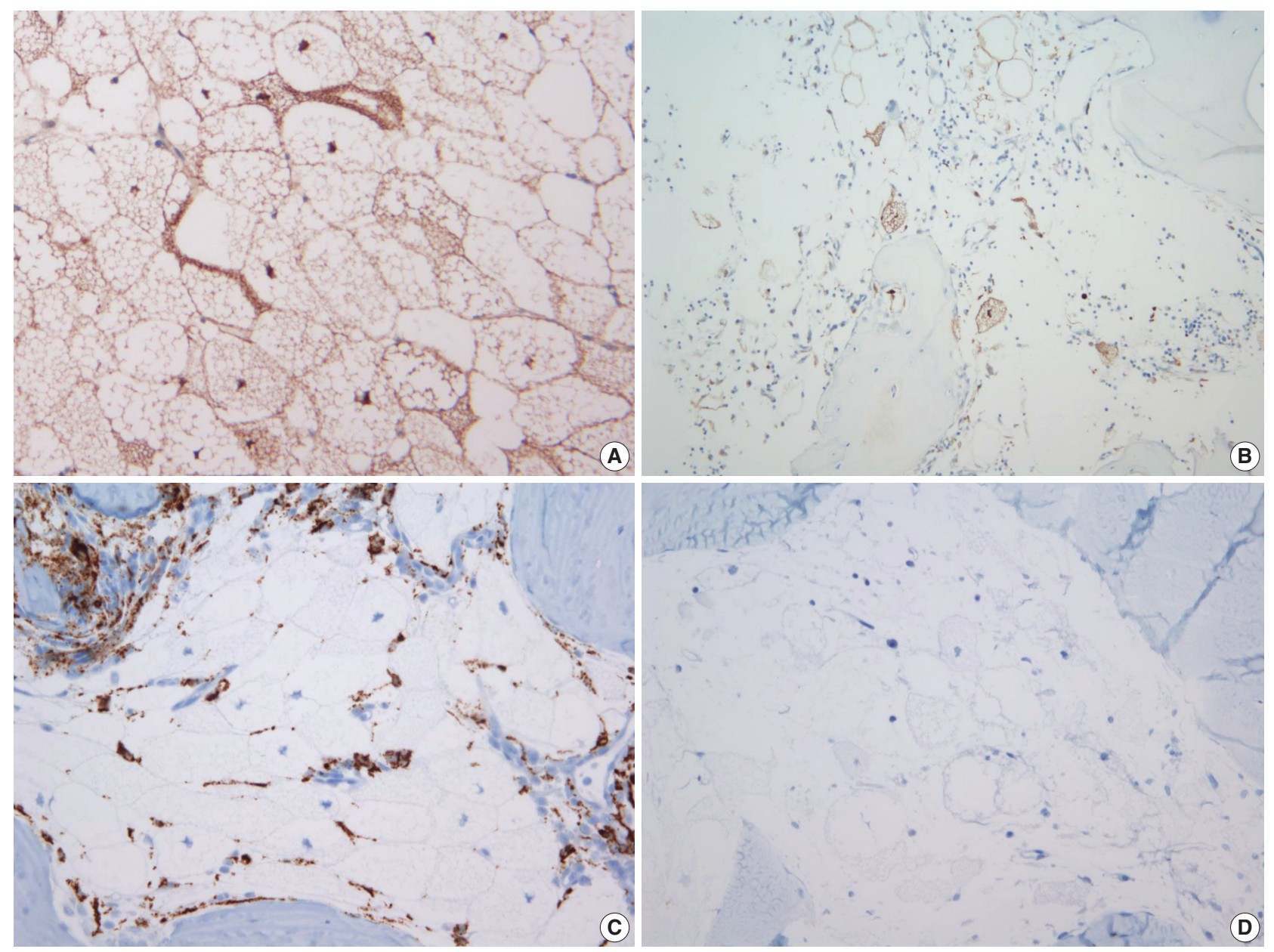

Fig. 3. Immunohistochemical stain of intraosseous hibernoma. (A) Immunohistochemical stain for S-100 protein shows diffuse positive staining in the nuclei and vacuolated cytoplasm. (B) S-100 protein immunohistochemistry highlights scattered brown fat cells intermingled with white adipose tissue. (C) CD68 staining are negative in brown fat cells. (D) Pan-cytokeratin also shows negative staining.

enhancement on post-contrast T1-weighted image. The lesions also revealed mildly increased uptake on PET scans and bone scans. The heterogeneous signals and contrast enhancement on $\mathrm{MRI}^{16,17}$ and increased uptake on bone scan ${ }^{8}$ are due to the increased vascularity within the lesions. Brown fat cells of hibernomas have numerous mitochondria in the cytoplasm. These mitochondria-rich brown fat cells present mild glucose hypermetabolism and increased ${ }^{18} \mathrm{~F}$ fluorodeoxyglucose uptake on PET/CT scans. ${ }^{18}$ Sclerosis on the $\mathrm{CT}$ scan is a nonspecific finding that may be seen in other lesions as a reactive change. Imaging findings of intraosseous hibernomas usually suggest a more common diagnosis of metastases and hemangiomas, not a hibernoma. ${ }^{5,7,8,10}$ The possibility of metastasis requires a histologic examination of the lesion to rule out malignancy. In our cohort, differential diagnoses for the imaging results were intraosseous hemangioma, bone marrow involvement of lymphoma, metastatic carcinoma, and osteomyelitis.

Microscopically, intraosseous hibernoma shows sheets and clusters of brown fat cells with voluminous multivacuolated cytoplasm and central small nuclei with minimal nuclear atypia. Unlike intraosseous lipomas, which destroy bony trabeculae and form a mass lesion, ${ }^{19}$ intraosseous hibernomas keep the bony trabeculae intact and infiltrate between them. ${ }^{4,7}$ This growth pattern is associated with reactive sclerosis of bony trabeculae affected by a lesion and was observed in most of the biopsied specimens of our cases. Sclerosis of bony trabeculae is a nonspecific finding that may be seen in other lesions that grow in the marrow space, including intraosseous hemangioma, lymphoma, and osteoblastic metastasis. ${ }^{5}$ Hibernoma cells with vacuolated cytoplasm may mimic lipoblasts of liposarcoma, foamy histiocytes, and granular cell tumors. Immunohistochemically, brown fat cells are positive for S-100 protein in the nuclei and cytoplasm and negative for CD68. Histiocytes can be distinguished by CD68 positivity. Lipoblasts are smaller and have fewer vacuoles than brown fat cells and show definite nuclear atypia. Absence of intracytoplasmic 
vacuoles can differentiate granular cell tumors from brown fat cells. In our cohort, there were misdiagnoses in two cases: one as lipoblasts in the marrow space, and the other as foamy histiocytes collection. The diagnosis of intraosseous hibernoma was confirmed by IHC testing for S-100 protein and CD68.

Osteoblasts and adipocytes are known to originate from common mesenchymal stem cells. ${ }^{20}$ Recently, it is known that differentiation of brown fat cells is associated with PRD1-BF1-RIZ1 homologous domain-containing 16 (PRDM16) under regulation by bone morphogenetic protein 7 (BMP7), which stimulates bone formation. ${ }^{21}$ Considering the function of BMP7 in bone formation and brown adipogenesis helps explain how brown fat cells and sclerotic bony trabeculae are mixed in intraosseous hibernomas.

Whether the brown fat cells in the marrow space are nonneoplastic resting cells or a neoplastic lesion is arguable. IHC staining does not help in this matter. In contrast to the intraosseous lipoma, which develops into a mass without hematopoietic marrow or bony trabeculae within the tumor, ${ }^{19}$ the fact that the intraosseous hibernoma is confined to the marrow spaces without forming a destructive mass suggests the former explanation. However, the radiologically distinct lesions described previously and in our studies support the lesion as being benign but neoplastic.

Intraosseous hibernomas are extremely rare and benign tumors. However, imaging findings for intraosseous hibernomas are nonspecific, and bone biopsy with histologic confirmation is usually required to distinguish it from other common conditions such as metastases. If pathologists do not consider the possibility of intraosseous hibernomas, the presence of brown fat cells in a bone biopsy specimen can be overlooked or misdiagnosed, leading to repeated biopsy to confirm the radiologically abnormal bone lesion. Therefore, pathologists need to be aware of intraosseous hibernomas to avoid missing this rare lesion.

\section{Conflicts of Interest}

No potential conflict of interest relevant to this article was reported.

\section{REFERENCES}

1. Furlong MA, Fanburg-Smith JC, Miettinen M. The morphologic spectrum of hibernoma: a clinicopathologic study of 170 cases. Am J Surg Pathol 2001; 25: 809-14.

2. Merkel H. On a pseudolipoma of the breast. Beitr Pathol Anat 1906; 39: 152-7.

3. Gery L. In discussion of MF Bonnel's paper. Bull Mem Soc Anat
(Paris) 1914; 89: 111-2.

4. Thorns C, Schardt C, Katenkamp D, Kähler C, Merz H, Feller AC. Hibernoma-like brown fat in the bone marrow: report of a unique case. Virchows Arch 2008; 452: 343-5.

5. Kumar R, Deaver MT, Czerniak BA, Madewell JE. Intraosseous hibernoma. Skeletal Radiol 2011; 40: 641-5.

6. Lynch DT, Dabney RS, Andrews JM. Intraosseous hibernoma or unusual location of brown fat? J Hematopathol 2013; 6: 151-3.

7. Bai S, Mies C, Stephenson J, Zhang PJ. Intraosseous hibernoma: a potential mimic of metastatic carcinoma. Ann Diagn Pathol 2013; 17: 204-6.

8. Botchu R, Puls F, Hock YL, et al. Intraosseous hibernoma: a case report and review of the literature. Skeletal Radiol 2013; 42: 1003-5.

9. Ringe KI, Rosenthal H, Langer F, Callies T, Wacker F, Raatschen HJ. Radiofrequency ablation of a rare case of an intraosseous hibernoma causing therapy-refractory pain. J Vasc Interv Radiol 2013; 24: 1754-6.

10. Bonar SF, Watson G, Gragnaniello C, Seex K, Magnussen J, Earwaker J. Intraosseous hibernoma: characterization of five cases and literature review. Skeletal Radiol 2014; 43: 939-46.

11. Dannheim K, Bhargava P. A rare finding of brown fat in bone marrow as a mimic for metastatic disease. Am J Hematol 2016; 91: 545-6.

12. Vlychou M, Teh J, Whitwell D, Athanasou NA. Intraosseous hibernoma: a rare adipocytic bone tumour. Skeletal Radiol 2016; 45: 1565-9.

13. Westacott L, Collins A, Dickenson I. Intraosseous hibernoma in the sacrum of an adult. Int J Surg Pathol 2016; 24: 749-52.

14. Himms-Hagen J. Brown adipose tissue thermogenesis: interdisciplinary studies. FASEB J 1990; 4: 2890-8.

15. van Marken Lichtenbelt WD, Vanhommerig JW, Smulders NM, et al. Cold-activated brown adipose tissue in healthy men. $\mathrm{N}$ Engl J Med 2009; 360: 1500-8.

16. Peer S, Kühberger R, Dessl A, Judmaier W. MR imaging findings in hibernoma. Skeletal Radiol 1997; 26: 507.

17. Lee JC, Gupta A, Saifuddin A, et al. Hibernoma: MRI features in eight consecutive cases. Clin Radiol 2006; 61: 1029-34.

18. Gaffney EF, Hargreaves HK, Semple E, Vellios F. Hibernoma: distinctive light and electron microscopic features and relationship to brown adipose tissue. Hum Pathol 1983; 14: 677-87.

19. Eyzaguirre E, Liqiang W, Karla GM, Rajendra K, Alberto A, Gatalica Z. Intraosseous lipoma: a clinical, radiologic, and pathologic study of 5 cases. Ann Diagn Pathol 2007; 11: 320-5.

20. Nombela-Arrieta C, Ritz J, Silberstein LE. The elusive nature and function of mesenchymal stem cells. Nat Rev Mol Cell Biol 2011; 12: 126-31.

21. Celi FS. Brown adipose tissue: when it pays to be inefficient. N Engl J Med 2009; 360: 1553-6. 